\title{
BMJ Wisconsin Card Sorting Test scores and open clinical and sociodemographic correlates in Schizophrenia: multiple logistic regression analysis
}

\author{
Masahiro Banno, ${ }^{1}$ Takayoshi Koide, ${ }^{1}$ Branko Aleksic, ${ }^{1}$ Takashi Okada, ${ }^{1}$ \\ Tsutomu Kikuchi, ${ }^{1,2}$ Kunihiro Kohmura, ${ }^{1}$ Yasunori Adachi, ${ }^{1}$ Naoko Kawano, ${ }^{1}$ \\ Tetsuya lidaka, ${ }^{1}$ Norio Ozaki ${ }^{1}$
}

To cite: Banno M, Koide T, Aleksic B, et al. Wisconsin Card Sorting Test scores and clinical and sociodemographic correlates in Schizophrenia: multiple logistic regression analysis. BMJ Open 2012;2: e001340. doi:10.1136/ bmjopen-2012-001340

- Prepublication history and additional material for this paper are available online. To view these files please visit the journal online (http://dx.doi.org/10.1136/ bmjopen-2012-001340).

Received 19 April 2012 Accepted 5 October 2012

Masahiro Banno and Takayoshi Koide contributed equally to this work.

This final article is available for use under the terms of the Creative Commons Attribution Non-Commercial 2.0 Licence; see http://bmjopen.bmj.com

${ }^{1}$ Department of Psychiatry, Nagoya University Graduate School of Medicine, Nagoya, Aichi-ken, Japan

${ }^{2}$ Department of Psychiatry, Matsuzaki Hospital,

Toyohashi, Aichi-ken, Japan

Correspondence to

Dr Takashi Okada;

okada@med.nagoya-u.ac.jp

\section{ABSTRACT}

Objectives: This study investigated what clinical and sociodemographic factors affected Wisconsin Card Sorting Test (WCST) factor scores of patients with schizophrenia to evaluate parameters or items of the WCST.

Design: Cross-sectional study.

Setting: Patients with schizophrenia from three hospitals participated.

Participants: Participants were recruited from July 2009 to August 2011. 131 Japanese patients with schizophrenia (84 men and 47 women, 43.5 \pm 13.8 years $($ mean $\pm S D)$ ) entered and completed the study. Participants were recruited in the study if they (1) met DSM-IV criteria for schizophrenia; (2) were physically healthy and (3) had no mood disorders, substance abuse, neurodevelopmental disorders, epilepsy or mental retardation. We examined their basic clinical and sociodemographic factors (sex, age, education years, age of onset, duration of illness, chlorpromazine equivalent doses and the positive and negative syndrome scale (PANSS) scores).

Primary and secondary outcome measures: All patients carried out the WCST Keio version. Five indicators were calculated, including categories achieved (CA), perseverative errors in Milner (PEM) and Nelson (PEN), total errors (TE) and difficulties of maintaining set (DMS). From the principal component analysis, we identified two factors (1 and 2). We assessed the relationship between these factor scores and clinical and sociodemographic factors, using multiple logistic regression analysis.

Results: Factor 1 was mainly composed of CA, PEM, PEN and TE. Factor 2 was mainly composed of DMS. The factor 1 score was affected by age, education years and the PANSS negative scale score. The factor 2 score was affected by duration of illness.

Conclusions: Age, education years, PANSS negative scale score and duration of illness affected WCST factor scores in patients with schizophrenia. Using WCST factor scores may reduce the possibility of type I errors due to multiple comparisons.

\section{ARTICLE SUMMARY}

Article focus

- To investigate relationships between Wisconsin Card Sorting Test (WCST) factor scores and clinical and sociodemographic factors in Japanese patients with schizophrenia using multiple logistic regression analysis.

- To show distribution of each WCST score for patients with schizophrenia.

Key messages

- Age, education years, positive and negative syndrome scale negative scale score and duration of illness affected two WCST factor scores.

- Using WCST factor scores may reduce the possibility of type I errors due to multiple comparisons.

Strengths and limitations of this study

- We conducted principal component analysis and identified two WCST factors. Components of two WCST factors in this study were similar to previous studies.

- This is the first study to investigate relationships between WCST factor scores and clinical and sociodemographic factors in patients with schizophrenia.

- We identified a clinical and sociodemographic factor (duration of illness) that affected the WCST factor 2 score. This is a new finding.

\section{INTRODUCTION}

Cognitive impairment in patients with schizophrenia has been evaluated as an indicator of outcome regarding social functioning and quality of life. ${ }^{12}$ It is reported that cognitive performance in patients with schizophrenia declines from prodrome to onset of schizophrenia (first episode). ${ }^{3}$ Moreover, it is reported that decline of cognitive performance exists before onset of schizophrenia. ${ }^{3}$ Many studies using brain imaging suggest that neurobiological changes in the brain are related to 
the cognitive impairment in schizophrenia. ${ }^{4-6}$ Therefore, some researchers regard cognitive impairment, rather than positive and negative symptoms, as the core pathology of schizophrenia. ${ }^{7}$

However, there are several problems when analysing cognitive impairment in schizophrenia. First, positive and negative syndromes modify cognitive performance. $^{8}{ }^{9}$ Second, intelligence level, intelligence profile (verbal IQ and performance IQ), and educational level could affect cognitive impairment in patients with schizophrenia. ${ }^{10-12}$ In brief, many factors have the potential to affect cognitive impairment in patients with schizophrenia. It is necessary to clarify the relationship between cognitive performance in patients with schizophrenia and clinical and sociodemographic factors in order to investigate what factors affect cognitive impairment in patients with schizophrenia.

Many neurocognitive tests have been used in order to evaluate cognitive performance in schizophrenia. The Wisconsin Card Sorting Test (WCST) is a neurocognitive test using cards and is one of the most frequently used executive function measures. ${ }^{13}$ A functional brain imaging study showed widespread activation across frontal and nonfrontal brain regions during WCST performance. ${ }^{14}$ It has been reported that each WCST score was related with social functioning in patients with schizophrenia. ${ }^{15-17}$

Recent reports suggest that WCST performance may decline during disease progression from prodrome to onset of schizophrenia. A steady (non-significant) progression of impairment on WCST perseverative errors (PE) was demonstrated from basic symptom at-risk (BS), ultra high-risk (UHR) and first-episode (FE) groups (BS: $\mathrm{z}=-0.74$; UHR: $\mathrm{z}=-0.88 ; \mathrm{FE}: \mathrm{z}=-0.97) .{ }^{3}$ However, negative and depressive symptoms may modify WCST performance in patients with schizophrenia, ${ }^{9} 18$ and many other factors (eg, premorbid IQ) may modify WCST scores. ${ }^{11}$

Factor structures of WCST in patients with schizophrenia have been investigated using principal component analysis and factor analysis of WCST scores. ${ }^{19-21}$ Differences in cognitive performance of WCST scores (categories achieved (CA) and PE) were shown between patients with schizophrenia and healthy controls (Cohens' $\mathrm{d}=0.91$ and 0.53 ) in one meta-analysis, but age, education years and other clinical and sociodemographic factors were not matched in the statistical analysis. ${ }^{10}$ In another previous study, age and education years affected CA and PE scores. ${ }^{22}$ In a different study, age affected PE score but education years did not affect either CA or PE scores. ${ }^{10}$ Additional two studies showed age of onset affected PE score ${ }^{23}$ and the positive and negative syndrome scale (PANSS) negative scale score affected CA score in patients with schizophrenia. ${ }^{9}$ These findings indicate that it is important to consider all clinical and sociodemographic factors to clarify which affect WCST scores in patients with schizophrenia.

In previous studies, the Wechsler Adult Intelligence Scale Full Scale IQ (FSIQ) showed significant correlations $(\mathrm{p}<0.05)$ with $\mathrm{CA}$, perseverative errors in Milner (PEM) and Nelson (PEN) and TE scores, while items 3 and 16 of the Brief Psychiatric Rating Scale showed significant correlations $(\mathrm{p}<0.05)$ with $\mathrm{CA}$, PEN and TE scores. ${ }^{24}$ Affective flattening and blunting and avolition-apathy on the Scale for the Assessment of Negative Symptoms showed significant correlations $(p<0.05)$ with CA, PEM, PEN, TE and difficulties of maintaining set (DMS) scores of Wisconsin Card Sorting Test Keio version (KWCST) in Japanese patients with schizophrenia $(\mathrm{n}=33) .{ }^{24}$ However, there is no previous study that investigated other clinical and sociodemographic factors (except IQ and negative symptoms) affecting KWCST scores. Therefore, we investigated clinical and sociodemographic factors affecting scores of $\mathrm{KWCST}^{25}$ (Japanese computerised version ${ }^{26}$ ) in Japanese patients with schizophrenia.

\section{METHODS AND PROCEDURES \\ Participants}

The study included 131 unrelated Japanese patients with schizophrenia (age 43.5 $\pm 13.8($ mean \pm SD), 84 men and 47 women) from three hospitals. The recruitment took place from both the outpatient department and the acute/ chronic wards in three hospitals. Fifty-one outpatients (15 acute phase patients and 36 chronic phase patients) and 55 inpatients ( 37 acute phase patients and 18 chronic phase patients) were recruited. Twenty-five patients were unspecified (outpatients or inpatients: 20 acute phase patients and 5 chronic phase patients). Participants were recruited from July 2009 to August 2011. Profiles of all the patients are shown in table 1. In total, 104 patients (78\%) were receiving concomitant medications, which could include benzodiazepines, barbiturates, anticholinergics, mood stabilisers and antidepressants.

This study protocol was approved by Nagoya University Graduate School of Medicine and Nagoya University

\begin{tabular}{lcl} 
Table 1 & Profiles of patients with schizophrenia \\
\hline & \multicolumn{3}{c}{$\begin{array}{l}\text { Patients with } \\
\text { schizophrenia }\end{array}$} \\
\cline { 2 - 3 } & $\mathbf{n}=\mathbf{1 3 1}$ Male & Female \\
\cline { 2 - 3 } Sex & $\mathbf{8 4}$ & $\mathbf{4 7}$ \\
\cline { 2 - 3 } & Average & (SD) \\
\hline Age (year) & 43.5 & $(13.8)$ \\
Education (year) & 12.4 & $(2.4)$ \\
Age of onset (year) & 26.3 & $(10.0)$ \\
Duration of illness (year) & 17.0 & $(12.8)$ \\
Chlorpromazine equivalent & 618.4 & $(391.1)$ \\
doses (mg) & & \\
PANSS scale & & \\
$\quad$ Positive (7-49) & 16.5 & $(5.3)$ \\
Negative (7-49) & 19.3 & $(5.6)$ \\
General (16-112) & 36.6 & $(9.4)$ \\
Total (30-210) & 72.4 & $(18.1)$ \\
\hline
\end{tabular}


Hospital Ethics Review Committee, and written informed consent was obtained from each participant. Participants were recruited for the study if they (1) met DSM-IV criteria for schizophrenia; (2) were physically healthy and (3) had no mood disorders, substance abuse, neurodevelopmental disorders, epilepsy or mental retardation. Consensus diagnoses were made by at least two experienced psychiatrists according to DSM-IV criteria on the basis of unstructured interviews with patients with schizophrenia (or their family members) and review of patients' medical records. Less than $5 \%$ of participants were excluded due to a lack of consensus. All subjects were unrelated to each other and lived in the central area of the mainland of Japan. A general characterisation and psychiatric assessment of the subjects is available elsewhere. ${ }^{27-29}$

\section{Measurement settings}

The WCST mainly assesses executive function, including cognitive flexibility in response to feedback..$^{30}$ KWCST is the Japanese version of the WCST modified by Kashima. ${ }^{25}$ KWCST consists of a card version and a computerised version, both of which have been used to investigate cognitive performance in patients with schizophrenia. ${ }^{31}{ }^{32}$ In KWCST, there are two levels of instruction. ${ }^{33}$ The subject is told that, at the first level, this is a test of classification based on any of the three categories of colour, shape or number, and that, at the second level, the tester's categories change when the subject continues to get correct answers at fixed times. The computerised version uses instruction through letters on the monitor and the synthetic sound of the computer in order to prevent potential bias derived from a confrontation test. We selected specific indicators (CA, PEM, PEN, TE and DMS) of KWCST in this analysis, given that these indicators were investigated in previous studies. ${ }^{31} \quad 32$ The computerised programme investigates these indicators at the second level only if the CA score at the first level is equal or less than 3. We got data for the following five indicators ${ }^{32} 34$ at the first and second levels in this study.

1. CA: the number of categories for which six consecutive correct responses are achieved (maximum CA is 8).

2. PEM: the number of incorrect responses in the same category as the immediately preceding correct response after the tester's categories change (maximum PEM is 47).

3. PEN: the number of incorrect responses in the same category as the immediately preceding incorrect response (maximum PEN is 47 ).

4. TE: the total number of incorrect responses (maximum TE is 48).

5. DMS: the number of times an incorrect response occurs after 2-5 consecutive correct responses (maximum DMS is 16).

We analysed KWCST (Japanese computerised version; ${ }^{26}$ Shimane University, Shimane, Japan) scores at the first level of the patients with schizophrenia.
Psychiatrists in three hospitals performed the KWCST assessment.

\section{Clinical and sociodemographic factors}

We investigated sex, age, education years, age of onset, duration of illness, chlorpromazine (CPZ) equivalent doses and PANSS scores as clinical and sociodemographic factors. Age was calculated based on the day we evaluated KWCST scores. Education years were calculated from elementary school entrance to the graduation or dropout of the last institution of higher education, which consisted of junior high school, senior high school, vocational school, junior college and university and graduate school. Age of onset was the age at onset of schizophrenia in each patient and was based on review of medical records. Duration of illness was defined from age of onset to age at the time of study. CPZ equivalent doses were the identified dose ratios of each antipsychotic in relation to $100 \mathrm{mg}$ of CPZ. ${ }^{35} \mathrm{CPZ}$ equivalent doses in this study were calculated based on the method by Inagaki and Inada. ${ }^{36}{ }^{37}$ PANSS is a standardised scale for evaluating positive and negative symptoms of schizophrenia and was used to evaluate severity of schizophrenia in the patients. ${ }^{38}$

\section{Statistical analysis}

Clinical profiles of the patients with schizophrenia are shown in table 1 . We investigated correlations of the five indicators of the KWCST (CA, PEM, PEN, TE and DMS) in patients with schizophrenia by Spearman's Rank Correlation Test.

\section{Principal component analysis}

The principal component model was based on Pearson's correlation matrix. We showed the Pearson's product moment correlation coefficients between the five indicators of WCST in supplementary table S1 (web-only file). WCST factors were identified by principal component analysis of the five indicators without rotation. Factors were retained using the eigenvalue $>1$ criterion.

\section{Main analysis}

In the main analysis, we investigated what clinical and sociodemographic factors affected WCST factor scores in a multiple logistic regression analysis. Our reasoning for not using multiple linear regression is explained in supplementary information S1 (web-only file). The dependent variables were WCST factor scores and independent variables were the following candidate clinical and sociodemographic factors: sex, age, education years, age of onset, duration of illness, CPZ equivalent doses and PANSS (positive, negative and general psychopathology scale) scores. We made a dummy conversion variable ( 1 or 0$)$ for sex. We converted factor scores into categorical variables ( 1 or 0$)$, using cut-off values that were median values of the factor scores. The median was chosen as a cut-off point for dependent variables based on reasons explained in supplementary information S2 
Figure 1 Distribution of Wisconsin Card Sorting Test scores in patients with schizophrenia $(n=131)$. None of the distribution was normal distribution. CA, categories achieved; DMS, difficulties of maintaining set; PEM, perseverative errors in Milner; PEN, perseverative errors in Nelson; TE, total errors;. (A) SD. (B) Percentage of cases.
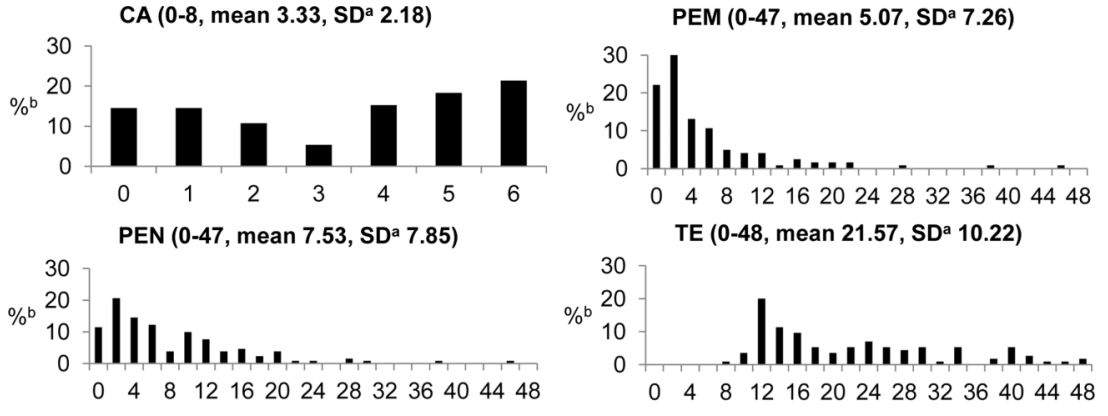

DMS (0-16, mean 1.27, SDa 1.67)

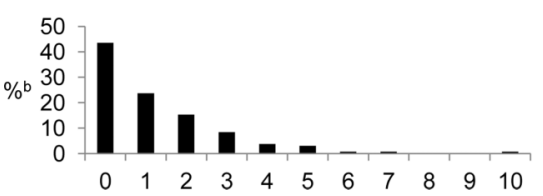

PEM (0-47, mean 5.07, SDa 7.26$)$ (web-only file). In our multiple logistic regression analysis, we did additional two tests. First, we did an omnibus test of model coefficients versus a model with intercept only. This test detects whether a model is significant $(p<0.05)$ or not; this is a test of the null hypothesis that adding any variables to the model has not significantly increased our ability to predict the dependent variable. A model is useless if the $p$ value in omnibus test was $>0.05$. Second, we did a Hosmer and Lemeshow goodness of fit test, which shows how well the model fits the data with $\mathrm{p}>0.05$ indicating good fit; this is a test of the null hypothesis that there is a linear relationship between the predictor variables and the log odds of the criterion variable. The hit rate in multiple logistic regression analysis is a measure how well a model predicts the dependent variable.

\section{Subanalysis}

In the subanalysis, we also investigated what clinical and sociodemographic factors affected the five indicators of WCST in the multiple logistic regression analysis. We used multiple logistic regression analysis in the subanalysis in order to compare the results between main and subanalysis. In this analysis, the dependent variables were the five indicators of WCST and independent variables were the candidate clinical and sociodemographic factors. We compared the results of the multiple logistic regression analysis with the results of previous studies. ${ }^{9} 1023$

\section{Software}

IBM SPSS statistical software (IBM Japan, Tokyo, Japan), V.19 was used for analyses. The significance level was set at $\mathrm{p}=0.05$ using a two-tailed t test.

\section{RESULTS}

Distribution of the WCST (CA, PEM, PEN, TE and DMS) scores in patients with schizophrenia is shown in figure 1. The numbers of patients in the following analyses were CA $n=131$, PEM n=122, PEN $n=131$, TE $\mathrm{n}=115$ and DMS $\mathrm{n}=131$ because of missing values in the data.

Spearman's rank correlation coefficients between the five indicators of WCST are shown in table 2. Although no strong correlation $(>0.8)$ was observed in any of these clinical and sociodemographic factors, the Spearman's correlation between PANSS negative scale score and PANSS general psychopathology scale score was high (0.74).

\section{Principal component analysis}

Two factors (1 and 2) were identified in principal component analysis of the five indicators of WCST. Factor 1 mainly consisted of CA, PEM, PEN and TE, and accounted for $65.6 \%$ of the total variance. Factor 2 mainly consisted of DMS and accounted for $23.2 \%$ of the total variance (table 3 and figure 2). We converted the factor 1 and factor 2 scores into categorical variables ( 1 or 0 ) using cut-off values. The cut-off values were the median values (factor 1:-0.299; factor 2:0.080). We used these categorical variables as dependent variables in multiple logistic regression analysis.

Table 2 Correlation coefficients for WCST scores in patients with schizophrenia

\begin{tabular}{lllllll}
\hline & \multicolumn{6}{c}{ Patients with schizophrenia (n=131) } \\
\cline { 3 - 7 } & & CA & PEM & PEN & TE & DMS \\
\hline Correlation & CA & - & - & - & - & - \\
coefficient† & PEM & $-0.70^{* *}$ & - & - & - & - \\
& PEN & $-0.79^{* *}$ & $0.73^{* *}$ & - & - & - \\
& TE & $-0.88^{* *}$ & $0.71^{* *}$ & $0.89^{* *}$ & - & - \\
& DMS & $-0.58^{* *}$ & $0.30^{*}$ & $0.28^{*}$ & $0.30^{*}$ & - \\
\hline${ }^{*} p<0.01$. \\
${ }^{* *} p<0.001$. \\
†Spearman's rank correlation coefficient. \\
CA, categories achieved; DMS, difficulties of maintaining set; PEM, \\
perseverative errors in Milner; PEN, perseverative errors in Nelson; \\
TE, total errors; WCST, Wisconsin Card Sorting Test.
\end{tabular}


Table 3 Factor loadings in principal component analysis in patients with schizophrenia $(n=131)$

\begin{tabular}{lcc} 
& Factor $\mathbf{1}$ & Factor $\mathbf{2}$ \\
\hline WCST score & & \\
CA & -0.89 & 0.36 \\
PEM & 0.84 & 0.27 \\
PEN & 0.92 & 0.27 \\
TE & 0.93 & 0.13 \\
DMS & 0.29 & -0.93 \\
Variance (\%) explained by each factor & 65.6 & 23.2 \\
Cumulative explained variance (\%) & 65.6 & 88.9 \\
\hline Factor analysis was based on principal component method \\
without rotation. & \\
Two factors were retained using the eigenvalue >1 criterion. \\
CA, categories achieved; DMS, difficulties of maintaining set; \\
PEM, perseverative errors in Milner; PEN, perseverative errors in \\
Nelson; TE, total errors; WCST, Wisconsin Card Sorting Test.
\end{tabular}

\section{Main analysis}

Age, education years and PANSS negative scale score significantly affected factor 1 score, and the duration of illness significantly affected factor 2 score in patients with schizophrenia (table 4). The details of the results from the multiple logistic regression analyses are shown in supplementary table S2 (web-only file). p Values in an omnibus test of model coefficients versus a model with intercept only were statistically significant $(\mathrm{p}<0.05)$ for all the models in WCST factor scores. In the Hosmer and Lemeshow goodness of fit test, all the models fit the data adequately with $\mathrm{p}>0.05$. Factor 1 score may be predicted precisely by this model considering hit rate $(0.77)$.

CPZ equivalent doses did not affect the WCST scores. PANSS positive scale score did not affect the WCST scores; whereas PANSS negative scale score did.

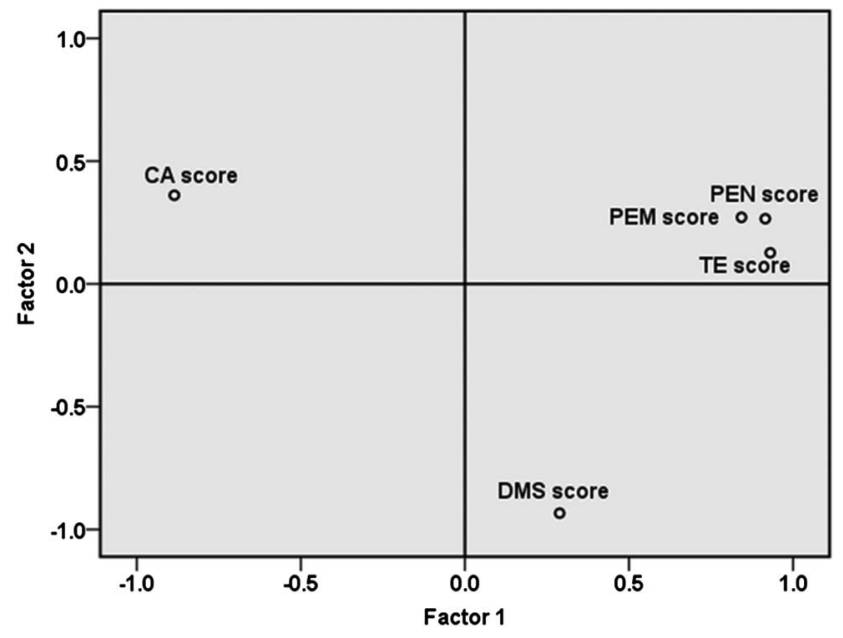

Figure 2 Component plot in principal component analysis of Wisconsin Card Sorting Test scores in patients with schizophrenia $(n=131)$. Abbreviations: $C A$, categories achieved; DMS, difficulties of maintaining set; PEM, perseverative errors in Milner; PEN, perseverative errors in Nelson; TE, total errors.

\section{Subanalysis}

In the subanalyses, age, education years and PANSS negative scale score significantly affected CA score. Age and education years significantly affected PEM, PEN and TE scores, and age significantly affected DMS score in patients with schizophrenia. The details of these results are shown in supplementary tables S3 and S4 (web-only file); supplementary table $\mathrm{S} 4$ includes the results of previous studies. $p$ Values in the omnibus test of model coefficients versus a model with intercept only were statistically significant $(p<0.05)$ for all the models for each WCST score, and all the models fit the data adequately in the Hosmer and Lemeshow goodness of fit test.

\section{DISCUSSION}

This study is the first to investigate the relationships between WCST factor scores and clinical and sociodemographic factors in Japanese patients with schizophrenia by multiple logistic regression analysis. We showed the distribution of each WCST score (figure 1). We conducted principal component analysis and identified two factors. The components of these two factors were similar to previous studies. ${ }^{19-21}$ Thus, we could reduce the number of WCST outcomes from five indicators to two factors (table 3). In assessment of cognitive function in patients with schizophrenia, using the WCST factor scores may reduce the possibility of type I errors due to multiple comparisons. We analysed the relationship between these two factors and clinical and sociodemographic factors with multiple logistic regression analysis. We found that age, education years, PANSS negative scale score and duration of illness affected the two WCST factor scores.

\section{Principal component analysis}

Our study showed that factor 1 mainly consisted of CA, PEM, PEN and TE and factor 2 mainly consisted of DMS. In the previous studies with principal component analysis and factor analysis of WCST scores in patients with schizophrenia, categories complete (CC; an indicator examining numbers of categories achieved in the same way as CA), PE (an indicator examining perseveration in the same way as PEM and PEN) and TE mainly constituted one factor. Failure to maintain set (FMS; an indicator examining difficulty of maintaining set, similar to DMS) mainly constituted another factor. ${ }^{19-21}$ Our results resembled the results of the principal component analysis and factor analysis of WCST in these previous studies. ${ }^{19-21}$

Factor 1, which included representative indicators (CC, PE, etc), was named as 'general executive functioning' in a previous study. ${ }^{21}$ Therefore, factor 1 in our study also may represent general executive functioning. In our study, factor 1 score showed a high contribution ratio of the total variance $(65.6 \%)$ in principal component analysis of WCST scores in patients with schizophrenia. WCST factor scores calculated by principal component analysis may be useful for reducing the 
Table 4 Clinical and sociodemographic factors for WCST scores of patients with schizophrenia in the current study (main analysis) and for previous studies

\begin{tabular}{|c|c|c|c|c|c|}
\hline & \multicolumn{5}{|c|}{ Patients with schizophrenia $(n=131)$} \\
\hline & \multicolumn{2}{|l|}{ Main analysis } & \multicolumn{3}{|c|}{ Previous studies } \\
\hline & Factor 1 score & Factor 2 score & CA† & PE† & TE† \\
\hline Sex & & & $\mathrm{n} / \mathrm{a}$ & $\mathrm{n} / \mathrm{a}$ & $\mathrm{n} / \mathrm{a}$ \\
\hline Age & $* * *$ & & nsł & $\bigcirc \ddagger$ & $\mathrm{n} / \mathrm{a}$ \\
\hline Education years & ** & & nsł & nsł & $\mathrm{n} / \mathrm{a}$ \\
\hline Age of onset & & & ns§ & ○ & $\mathrm{n} / \mathrm{a}$ \\
\hline Duration of illness & & * & nsł & nsł & $\mathrm{n} / \mathrm{a}$ \\
\hline Chlorpromazine equivalent doses & & & $\mathrm{n} / \mathrm{a}$ & $\mathrm{n} / \mathrm{a}$ & $\mathrm{n} / \mathrm{a}$ \\
\hline \multicolumn{6}{|l|}{ PANSS score } \\
\hline Positive (7-49) & & & nsף & $\mathrm{n} / \mathrm{a}$ & $\mathrm{n} / \mathrm{a}$ \\
\hline Negative (7-49) & * & & ○ा & $\mathrm{n} / \mathrm{a}$ & $\mathrm{n} / \mathrm{a}$ \\
\hline General (16-112) & & & ns & $\mathrm{n} / \mathrm{a}$ & $\mathrm{n} / \mathrm{a}$ \\
\hline Hit rate & 0.77 & 0.58 & $\mathrm{n} / \mathrm{a}$ & $\mathrm{n} / \mathrm{a}$ & $\mathrm{n} / \mathrm{a}$ \\
\hline \multicolumn{6}{|c|}{$\begin{array}{l}{ }^{*} \mathrm{p}<0.05 . \\
{ }^{*} \mathrm{p}<0.01 . \\
{ }^{* *} \mathrm{p}<0.001 . \\
+\mathrm{CA}, \mathrm{PE} \text { and TE were included in factor } 1 \text { in a previous study. } \\
\text { †Reference } 10 . \\
\text { §Reference } 23 . \\
\text { TReference } 9 . \\
\text { CA, categories achieved; DMS, difficulties of maintaining set; } \mathrm{n} / \mathrm{a} \text {, data not available; ns, not significant; PANSS, positive and negative } \\
\text { syndrome scale; PEM, perseverative errors in Milner; PEN, perseverative errors in Nelson; TE, total errors; WCST, Wisconsin Card Sorting } \\
\text { Test. }\end{array}$} \\
\hline
\end{tabular}

possibility of type I errors due to multiple comparisons. Factors 1 and 2 in our study resembled those in previous studies. ${ }^{19-21}$ Therefore, the KWCST measures cognitive function similarly to the traditional WCST.

We compared the Spearman's rank correlation coefficients with the Pearson's product moment correlation coefficients between the five indicators of WCST (table 2 and supplementary table S1). Correlations between CA, PEM, PEN and TE and a correlation between CA and DMS were statistically significant $(p<0.001)$. In this point, both correlation coefficients showed the same direction. Therefore, using Pearson's correlation matrix, instead of Spearman's correlation matrix, in principal component analysis may be justified in our study.

\section{Main analysis}

We identified clinical and sociodemographic factors (age, education years and PANSS negative scale score) affecting WCST factor 1 score. We also identified a clinical and sociodemographic factor (duration of illness) affecting WCST factor 2 score. This is an important new finding. Comparing the three main previous studies 91023 with the current study, we summarised shared and different findings, shown in table 4.

The shared findings were that age and PANSS negative scale score were related to WCST scores (table 4). 91023

Two findings differed from previous studies (table 4). ${ }^{9} \quad 10 \quad 23$ First, we found a new relationship between education years and WCST scores. Second, we found no relationship between age of onset and WCST scores. Differences in the results between previous studies 91023 and our study may be explained by differences of ethnicity, distribution of age and education years, types of statistical analysis used, and the version of WCST. These differences suggest that future studies about WCST should be conducted with attention to these conditions.

CPZ equivalent doses did not affect the WCST scores in this study. This result was in the same direction as one meta-analysis $(n=4524)$ though recent studies had suggested the possibility of an effect. ${ }^{31} 3940$ Future studies will be necessary to clarify whether CPZ equivalent doses affect WCST scores under other conditions.

PANSS positive scale score did not affect the WCST scores but the PANSS negative scale score did. A recent meta-analysis $(n=6519)$ suggested that negative symptoms related to cognitive performance in patients with schizophrenia whereas positive symptoms did not. ${ }^{41}$ This suggests that the relationships between PANSS positive and negative scale scores and WCST scores in this study may be reasonable.

\section{Subanalysis}

We found that factor 1 score and factor 1 score's main components (CA, PEM, PEN and TE) related to age and education years (see online supplementary table S5 (web-only file)).

The effect of duration of illness on WCST factor 2 score, which was mainly influenced by DMS, is the novel finding of the main analysis. However, DMS is not significantly associated with the duration of illness in the subanalysis (see online supplementary table S5 (web-only file)). This 
discrepancy between the main analysis and subanalysis may be derived from the difference between DMS and factor 2 (factor 2 included not only DMS, but also CA, PEM, PEN and TE).

\section{Limitations}

There are several limitations in this study. First, other clinical and sociodemographic factors that were not investigated in the current study could affect WCST scores. Candidates for such clinical and sociodemographic factors are $\mathrm{IQ}^{42}$ participants' dominant arm, experience with using a computer, doses of drugs affecting cognitive performance (anticholinergics, benzodiazepines, etc), sleep, ${ }^{43}$ eating and risk factors of arteriosclerosis (body mass index, blood pressure, etc). ${ }^{44}$ It may be useful to include these factors in future studies. Second, the WCST indicators (CA, PEM, PEN, TE and DMS scores) in our study did not cover all WCST indicators; we selected the major five indicators. We might find other factors by principal component analysis or new relationships between new WCST factors and clinical and sociodemographic factors if we included other clinical indicators. Third, instead of using Spearman's correlation matrix in the principal component analysis, which might be more appropriate method in terms of the non-normal distribution of five WCST indicators, we used Pearson's correlation matrix. Fourth, we dichotomised continuous variables (WCST factor scores) in the multiple logistic regression analysis. Therefore, careful interpretation of the results may be needed, considering the statistical weak points. ${ }^{45}$

\section{CONCLUSION}

This study is the first study that investigated clinical and sociodemographic factors affecting WCST factor scores calculated by principal component analysis in patients with schizophrenia. The study was conducted in a relatively large Japanese population. We showed distribution of measured five WCST indicators in patients with schizophrenia and confirmed two WCST factors by principal component analysis. Age, education years, PANSS negative scale score and duration of illness affected WCST scores in patients with schizophrenia. The interaction between the duration of illness and a factor of the WCST needs further confirmation in future studies because there was a discrepancy between the results of the main analysis and the subanalysis in this study.

Acknowledgements We sincerely thank the patients for their participation in this study. We express our gratitude to Ryoko Ishihara PhD, Hiromi Noma, Saori Yamashita PhD, Mami Yoshida and Yoshihito Ito MD, PhD for their technical assistance.

Contributors MB, TKo and NO conceived and designed the experiments. MB, TKo, TKi, KK and YA performed the experiments. MB, TKo, BA, TO, NK, TI and NO analysed the data. MB, TKo and YA contributed reagents/ materials/analysis tools. MB, TKo, TO, BA and NO wrote the paper.

Funding This work was supported by research grants from the Ministry of Education, Culture, Sports, Science and Technology of Japan; the Ministry of
Health, Labor and Welfare of Japan; Grant-in-Aid for 'Integrated research on neuropsychiatric disorders' carried out under the Strategic Research Program for Brain Sciences by the Ministry of Education, Culture, Sports, Science and Technology of Japan; Grant-in-Aid for Scientific Research on Innovative Areas (Comprehensive Brain Science Network) from the Ministry of Education, Science, Sports and Culture of Japan; The Academic Frontier Project for Private Universities, Comparative Cognitive Science Institutes, Meijo University and the Core Research for Evolutional Science and Technology. The funders had no role in study design, data collection and analysis, decision to publish or preparation of the manuscript.

Competing interests None.

Ethics approval This study was approved under the guidelines for epidemiological studies by the Nagoya University Graduate School of Medicine and Nagoya University Hospital Ethics Review Committee and was conducted in accordance with the Helsinki Declaration. Written informed consent was obtained from each subject before the start of the study.

Provenance and peer review Not commissioned; externally peer reviewed.

Data sharing statement No additional data are available.

\section{REFERENCES}

1. Green MF, Kern RS, Braff DL, et al. Neurocognitive deficits and functional outcome in schizophrenia: are we measuring the 'right stuff'? Schizophr Bull 2000;26:119-36.

2. Fett AK, Viechtbauer W, Dominguez MD, et al. The relationship between neurocognition and social cognition with functional outcomes in schizophrenia: a meta-analysis. Neurosci Biobehav Rev 2011;35:573-88.

3. Simon AE, Cattapan-Ludewig K, Zmilacher S, et al. Cognitive functioning in the schizophrenia prodrome. Schizophr Bull 2007;33:761-71.

4. Antonova E, Sharma T, Morris R, et al. The relationship between brain structure and neurocognition in schizophrenia: a selective review. Schizophr Res 2004;70:117-45.

5. Bartzokis G, Lu PH, Amar CP, et al. Long acting injection versus oral risperidone in first-episode schizophrenia: differential impact on white matter myelination trajectory. Schizophr Res 2011;132:35-41.

6. Ehrlich S, Brauns S, Yendiki A, et al. Associations of cortical thickness and cognition in patients with Schizophrenia and healthy controls. Schizophr Bull 2011;38:1050-62.

7. Young JW, Powell SB, Risbrough V, et al. Using the MATRICS to guide development of a preclinical cognitive test battery for research in schizophrenia. Pharmacol Ther 2009;122:150-202.

8. Villalta-Gil V, Vilaplana M, Ochoa S, et al. Neurocognitive performance and negative symptoms: are they equal in explaining disability in schizophrenia outpatients? Schizophr Res 2006;87:246-53.

9. Rodriguez-Jimenez R, Aragües M, Jimenez-Arriero MA, et al Psychopathology and Wisconsin Card Sorting Test performance in male schizophrenic patients: influence of dual diagnosis. Psychopathology 2008;41:58-64.

10. Laws KR. A meta-analytic review of Wisconsin Card Sort studies in schizophrenia: general intellectual deficit in disguise? Cognit Neuropsychiatry 1999;4:1-30; discussion 31-5.

11. Fuller R, Nopoulos P, Arndt S, et al. Longitudinal assessment of premorbid cognitive functioning in patients with schizophrenia through examination of standardized scholastic test performance. Am J Psychiatry 2002;159:1183-9.

12. Irani F, Kalkstein S, Moberg EA, et al. Neuropsychological performance in older patients with schizophrenia: a meta-analysis of cross-sectional and longitudinal studies. Schizophr Bull 2011;37:1318-26.

13. Alvarez JA, Emory E. Executive function and the frontal lobes: a meta-analytic review. Neuropsychol Rev 2006;16:17-42.

14. Nyhus E, Barceló F. The Wisconsin Card Sorting Test and the cognitive assessment of prefrontal executive functions: a critical update. Brain Cogn 2009;71:437-51.

15. Penades R, Boget T, Catalan R, et al. Cognitive mechanisms, psychosocial functioning, and neurocognitive rehabilitation in schizophrenia. Schizophr Res 2003;63:219-27.

16. Bowie CR, Leung WW, Reichenberg A, et al. Predicting schizophrenia patients' real-world behavior with specific neuropsychological and functional capacity measures. Biol Psychiatry 2008;63:505-11. 
17. Zayat E, Rempfer M, Gajewski B, et al. Patterns of association between performance in a natural environment and measures of executive function in people with schizophrenia. Psychiatry Res 2011;187:1-5.

18. Tomida K, Takahashi N, Saito S, et al. Relationship of psychopathological symptoms and cognitive function to subjective quality of life in patients with chronic schizophrenia. Psychiatry Clin Neurosci 2010;64:62-9.

19. Cuesta MJ, Peralta V, Caro F, et al. Schizophrenic syndrome and Wisconsin Card Sorting Test dimensions. Psychiatry Res 1995;58:45-51.

20. Koren D, Seidman LJ, Harrison RH, et al. Factor structure of the Wisconsin Card Sorting Test: dimensions of deficit in schizophrenia. Neuropsychology 1998;12:289-302.

21. Polgár P, Réthelyi JM, Bálint $\mathrm{S}$, et al. Executive function in deficit schizophrenia: what do the dimensions of the Wisconsin Card Sorting Test tell us? Schizophr Res 2010;122:85-93.

22. Rhodes MG. Age-related differences in performance on the Wisconsin Card Sorting Test: a meta-analytic review. Psychol Aging 2004;19:482-94.

23. Bellino S, Rocca P, Patria L, et al. Relationships of age at onset with clinical features and cognitive functions in a sample of schizophrenia patients. J Clin Psychiatry 2004;65:908-14.

24. Kashima $\mathrm{H}$, Kato $\mathrm{M}$, Handa $\mathrm{T}$. Neuropsychological studies on frontal lobe function of the patients with chronic schizophrenia. Results of application of the new modified Wisconsin Card Sorting Test. Rinsho Seishin Igaku (Japan J Clin Psychiatry) 1985;14:1479-89.

25. Kashima H, Handa T, Kato M, et al. Neuropsychological investigation on chronic schizophrenia. Aspects of its frontal functions. In: Takahashi R, Flor-Henry P, Gruzelier , et al., eds. Cerebral dynamics, laterality and psychopathology. 1st edn. Amsterdam: Elsevier, 1987:337-45.

26. Kobayashi S. Wisconsin Card Sorting Test Program Keio-F-S-version (Web site, in Japanese). 1999 1999. http://cvddb.med.shimane-u.ac.jp/ cvddb/user/wisconsin.htm(accessed 8 Sep 2012).

27. Aleksic B, Kushima I, Ito Y, et al. Genetic association study of KREMEN1 and DKK1 and schizophrenia in a Japanese population. Schizophr Res 2010;118:113-17.

28. Koide $\mathrm{T}$, Aleksic B, Ito $\mathrm{Y}$, et al. A two-stage case-control association study of the dihydropyrimidinase-like 2 gene (DPYSL2) with schizophrenia in Japanese subjects. J Hum Genet 2010;55:469-72.

29. Banno M, Koide T, Aleksic B, et al. A case control association study and cognitive function analysis of neuropilin and tolloid-like 1 gene and schizophrenia in the Japanese population. PLoS One 2011;6: e28929.

30. Heaton RK. The wisconsin card sorting test (manual). Odessa, FL: Psychological Assessment Resources, 1981.
31. Hori $\mathrm{H}$, Noguchi $\mathrm{H}$, Hashimoto R, et al. Antipsychotic medication and cognitive function in schizophrenia. Schizophr Res 2006;86:138-46.

32. Suzuki H, Gen K, Inoue Y. An unblinded comparison of the clinical and cognitive effects of switching from first-generation antipsychotics to aripiprazole, perospirone or olanzapine in patients with chronic schizophrenia. Prog Neuro-Psychopharmacol Biol Psychiatry 2011;35:161-8.

33. Kashima H. Tests for executive function. Seishinka Shindangaku. Arch Psychiatric Diagn Clin Eval 2002;13:205-12.

34. Heaton RK, Chelune GL, Talley JL, et al. Wisconsin Card Sorting Test manual: revised and expanded. Oclessa, FL: Psychological Assessment Resourses, 1993.

35. Andreasen NC, Pressler M, Nopoulos P, et al. Antipsychotic dose equivalents and dose-years: a standardized method for comparing exposure to different drugs. Biol Psychiatry 2010;67:255-62.

36. Inagaki A, Inada T. Dose equivalence of psychotropic drugs. Part XXI: dose equivalence of novel antipsychotics: Blonanserin. Rinsho Seishin Yakuri (Japn J Clin Psychopharmacol) 2008;11:887-90.

37. Inagaki A, Inada T. Dose equivalence of psychotropic drugs. Part XXII: dose equivalence of depot antipsychotics III: risperidon long-acting injection. Rinsho Seishin Yakuri (Japn J Clin Psychopharmacol) 2010;13:1349-53.

38. Kay SR, Fiszbein A, Opler LA. The positive and negative syndrome scale (PANSS) for schizophrenia. Schizophr Bull 1987;13:261-76.

39. Johnson-Selfridge M, Zalewski C. Moderator variables of executive functioning in schizophrenia: meta-analytic findings. Schizophr Bull 2001;27:305-16.

40. Kawai N, Yamakawa Y, Baba A, et al. High-dose of multiple antipsychotics and cognitive function in schizophrenia: the effect of dose-reduction. Prog Neuro-Psychopharmacol Biol Psychiatry 2006;30:1009-14.

41. Ventura J, Hellemann GS, Thames AD, et al. Symptoms as mediators of the relationship between neurocognition and functional outcome in schizophrenia: a meta-analysis. Schizophr Res 2009;113:189-99.

42. Golden CJ, Kushner T, Lee B, et al. Searching for the meaning of the category test and the Wisconsin Card Sort Test: a comparative analysis. Int J Neurosci 1998;93:141-50.

43. Killgore WD, Kahn-Greene ET, Grugle NL, et al. Sustaining executive functions during sleep deprivation: a comparison of caffeine, dextroamphetamine, and modafinil. Sleep 2009;32:205-16.

44. Friedman JI, Wallenstein S, Moshier E, et al. The effects of hypertension and body mass index on cognition in schizophrenia. Am J Psychiatry 2010;167:1232-9.

45. Altman DG, Royston P. The cost of dichotomising continuous variables. BMJ 2006;332:1080. 